\title{
Spin excitations in cubic maghemite nanoparticles studied by time-of-flight neutron spectroscopy
}

\author{
S. Disch, ${ }^{1,2, *}$ R. P. Hermann, ${ }^{1,3}$ E. Wetterskog, ${ }^{4}$ A. A. Podlesnyak,${ }^{5}$ K. An, ${ }^{6,7}$ T. Hyeon, ${ }^{6,7}$ \\ G. Salazar-Alvarez, ${ }^{4}$ L. Bergström, ${ }^{4}$ and Th. Brückel ${ }^{1, \dagger}$ \\ ${ }^{1}$ Jülich Centre for Neutron Science JCNS and Peter Grünberg Institut PGI, JARA-FIT, Forschungszentrum Jülich, D-52425 Jülich, Germany \\ ${ }^{2}$ Institut Laue-Langevin, F-38042 Grenoble, France \\ ${ }^{3}$ Faculty of Science, University of Liège, B-4000 Liège, Belgium \\ ${ }^{4}$ Department of Materials and Environmental Chemistry, Arrhenius Laboratory, Stockholm University, S-10691 Stockholm, Sweden \\ ${ }^{5}$ Quantum Condensed Matter Division, Oak Ridge National Laboratory, Oak Ridge, Tennessee 37831, USA \\ ${ }^{6}$ Center for Nanoparticle Research, Institute for Basic Science (IBS), Seoul 151-742, Korea \\ ${ }^{7}$ School of Chemical and Biological Engineering, Seoul National University, Seoul 151-742, Korea \\ (Received 2 December 2013; revised manuscript received 18 January 2014; published 5 February 2014)
}

\begin{abstract}
We have determined the field dependence of collective magnetic excitations in iron oxide nanoparticles of cubic shape with 8.42(2) nm edge length and a narrow log normal size distribution of 8.2(2)\% using time-of-flight neutron spectroscopy. The energy dependence of the uniform precession modes was investigated up to $5 \mathrm{~T}$ applied field and yields a Landé factor $g=2.05(2)$ as expected for maghemite $\left(\gamma-\mathrm{Fe}_{2} \mathrm{O}_{3}\right)$ nanoparticles. A large effective anisotropy field of $B_{A, \text { eff }}=0.45(16) \mathrm{T}$ was determined, in excellent agreement with macroscopic measurements. This anisotropy is attributed to enhanced shape anisotropy in these monodisperse cubic nanoparticles. The combination of our results with macroscopic magnetization information provides a consistent view of the energy scales of superparamagnetic relaxation and collective magnetic excitations in magnetic nanoparticles.
\end{abstract}

DOI: 10.1103/PhysRevB.89.064402

PACS number(s): 75.75.Jn, 75.30.Gw, 78.70.Nx

\section{INTRODUCTION}

Magnetic nanoparticles reveal unique magnetic properties which make them interesting for applications in data storage [1], electronic and mechanical engineering [2-4], and biomedical applications [5]. Magnetic properties and in particular the magnetization dynamics in nanoparticles are significantly distinct from the bulk properties [6]. The magnetic anisotropy energy scales with the particle volume and can become equivalent or smaller than the thermal energy for small nanoparticles, leading to superparamagnetic relaxation which has been found important for biomedical applications including medical imaging [5,7,8], drug delivery [5], or hyperthermia [5]. Below the superparamagnetic transition temperature (blocking temperature), the magnetization direction is not fixed but still fluctuates close to the easy direction of the material. These thermally excited precession modes and the transitions between different precession states of different precession angles have been termed collective magnetic excitations [9-11]. As opposed to spin waves in the macroscopic material, such uniform excitations are dominant for nanoparticles with uniaxial anisotropy at low temperatures and can be described by spin wave modes with the wave vector $Q=0[10]$.

Conventional techniques for the study of uniform excitations in magnetic materials include ac and $\mathrm{dc}$ magnetometry as well as Mössbauer spectroscopy, where collective magnetic excitations have been observed as reduction of the average magnetization and the magnetic hyperfine splitting $[9,12]$. For measurements of the excitation energy $\Delta E$ of the uniform precession states, inelastic neutron scattering techniques give access to the relevant time scales in

\footnotetext{
*Present address: Department Chemie, Universität zu Köln, Luxemburger Strasse 116, 50939 Köln, Germany.

†T.Brueckel@fz-juelich.de
}

the order of $10^{-12}-10^{-7} \mathrm{~s}$. The collective magnetic excitations in antiferromagnetic and ferromagnetic nanoparticle systems and their dependence on the applied temperature and magnetic field have been intensely studied using inelastic neutron scattering [13-19]. Whereas most of the previous studies of collective magnetic excitations in magnetic nanoparticles have been carried out using triple-axis neutron spectroscopy in the vicinity of the magnetic Bragg reflections, magnetic excitations in nanoparticles have recently also been studied using time-of-flight neutron spectroscopy [20].

Here we present a neutron time-of-flight study of the magnetization dynamics in monodisperse maghemite $\left(\gamma-\mathrm{Fe}_{2} \mathrm{O}_{3}\right)$ nanoparticles. Particular emphasis is on the morphological precharacterization confirming the narrow size distribution and defined particle shape. Field dependent inelastic neutron scattering data gives access to the collective magnetic excitations observed as satellite peaks around the ferrimagnetic (111) reflection. The magnetic anisotropy constant is derived through the obtained effective anisotropy field and related to results of macroscopic magnetization measurements in combination with morphological characterization.

\section{EXPERIMENT}

\section{A. Synthesis}

Iron oxide nanocubes were prepared according to a thermolytic approach involving thermal decomposition of an iron oleate precursor as described previously [21-24]. The obtained nanoparticles are capped by an oleic acid ligand layer in order to prevent agglomeration. For minimization of incoherent and inelastic scattering contributions of this nondeuterated organic compound, the sample was subjected to several cycles of precipitation and redispersion, yielding a pasty nanoparticle sample with an organic content as low as $12 \mathrm{wt}$ \%. In addition, monodispersed iron oxide nanoparticles with a diameter of 14.6(2) $\mathrm{nm}$ were prepared as previously described [24-26]. 


\section{B. Morphological characterization}

Small-angle x-ray scattering (SAXS) was performed at the B1 beamline of HASYLAB/DESY. Dilute nanoparticle dispersions in toluene were measured using an incident $\mathrm{X}$-ray energy of $12 \mathrm{keV}$ and detector distances of 0.93 and $3.63 \mathrm{~m}$. Data was recorded using a Pilatus $300 \mathrm{~K}$ detector and averaged circularly. Small-angle neutron scattering (SANS) was measured at the D22 instrument at ILL, using $6 \AA$ neutron wavelength and detector distances of 2 and $8 \mathrm{~m}$ with a constant collimation distance of $8 \mathrm{~m}$.

Transmission electron microscopy (TEM) images were obtained using a JEOL JEM-2100 microscope equipped with a $\mathrm{LaB}_{6}$ filament operated at $200 \mathrm{kV}(f=2.7 \mathrm{~mm}, \mathrm{Cs}=$ $1.4 \mathrm{~mm}, \mathrm{Cc}=1.8 \mathrm{~mm}$, and point resolution $=2.5 \AA$ ).

\section{Structural characterization}

Synchrotron x-ray powder diffraction (XRPD) was measured at the 6-ID-D high energy beamline at the Advanced Photon Source (APS) of Argonne National Laboratory, IL, USA. A powder sample of nanoparticles was measured using an incident energy of $99.88 \mathrm{keV}$, corresponding to an $\mathrm{x}$-ray wavelength of $0.1243 \AA$. Data were collected using an image plate detector (Mar345) at a distance of $1.601 \mathrm{~m}$ from the sample, calibrated with NIST 640c Si. TOF neutron powder diffraction was measured at POWGEN at SNS, ORNL [27] using two different time frames corresponding to wavelength bands of $0<\lambda<1.066 \AA$ and $3.198 \AA$. Both X-ray and neutron diffraction data were refined according to the Le Bail approach using the GSAS and EXPGUI software package [28,29]. The diffuse scattering background, which is particularly strong in the neutron diffraction data due to the undeuterated oleic acid ligand shell of the particles, was treated using a shifted Chebyshev polynomial of 12 parameters. Polarized neutron diffraction with full $x y z$ polarization analysis was carried out on the powder nanoparticle sample of larger particle size (14.6 nm diameter) at the DNS instrument of JCNS, Garching, Germany, using an incident neutron wavelength of $4.75 \AA$. $\mathrm{NiCr}$ and vanadium samples were measured as references, and the nuclear coherent, nuclear incoherent, and magnetic scattering contributions were separated.

\section{Macroscopic magnetization}

Temperature dependent magnetization measurements were performed using a Quantum Design MPMS XL SQUID. A liquid 0.03 wt. \% dispersion of nanoparticles in toluene was measured using an airtight Vespel polyimide sample holder of $38.5 \mu \mathrm{l}$ sample volume. Magnetization measurements were carried out between 5 and $300 \mathrm{~K}$ with a heating rate of $1 \mathrm{~K} / \mathrm{min}$ in an applied magnetic field of $\mu_{0} H=5 \mathrm{mT}$, after cooling in either applied field or in zero field. The blocking temperature was determined by fitting a Lorentzian with a linear background to the ZFC data. Field dependent magnetization measurements were performed using a Quantum Design Physical Property Measuring System (PPMS-9) equipped with a superconducting magnet and a vibrating sample magnetometer (VSM) option. The magnetization of a 0.1 wt. $\%$ dispersion of the nanoparticles in paraffin was measured up to a maximum field $\mu_{0} H=1.5 \mathrm{~T}$. The data was fitted with the Langevin equation, including an excess susceptibility term accounting for a diamagnetic contribution.

\section{E. Inelastic neutron scattering}

Inelastic neutron scattering experiments were performed at the Cold Neutron Chopper Spectrometer (CNCS) at SNS, ORNL [30]. An incident neutron energy of $2.5 \mathrm{meV}$ was chosen, providing an elastic energy resolution of $0.06 \mathrm{meV}$ determined from vanadium scattering. Measurements were performed at $100 \mathrm{~K}$ in applied magnetic fields up to $\mu_{0} H=5 \mathrm{~T}$ with exposure times of $3 \mathrm{~h}$ up to $22 \mathrm{~h}$, in detail: $5 \mathrm{~T}(22 \mathrm{~h})$, $4 \mathrm{~T}(9 \mathrm{~h}), 3 \mathrm{~T}(5 \mathrm{~h}), 2 \mathrm{~T}(5 \mathrm{~h}), 1 \mathrm{~T}(3 \mathrm{~h})$, and $0 \mathrm{~T}(19 \mathrm{~h})$. The measured data was reduced using MANTIDPLOT software [31]. Correction for $k_{i} / k_{f}$ was included for all measurements, and detector efficiency correction using a vanadium reference sample was performed for the elastic line cuts, but omitted for display of the inelastic scattering data for statistical reasons. The $S(Q, \omega)$ data was rebinned using $0.02 \AA^{-1}$ and $0.02 \mathrm{meV}$ step sizes. Inelastic cuts were determined using a step size of $0.02 \mathrm{meV}$ and a projection in the $Q$ range of $1.24-1.36 \AA^{-1}$ for evaluation of the magnetic excitations and using a $Q$ range of 1.0-1.2 $\AA^{-1}$ for determination of the background scattering contribution. From the latter, a phenomenological scattering background was determined consisting of a polynomial and a quasielastic Gaussian contribution. For refinement of the inelastic magnetic excitation peaks of the (111) maghemite reflection, the predetermined background function was used along with Gaussian contributions for the elastic line, an additional quasielastic scattering contribution, as well as the inelastic satellite peaks. In order to enhance the reliability of the fit of the inelastic satellite peak positions, we constrained their energy position to be equal on both sides of the elastic line and their peak width to be equal to the elastic linewidth.

\section{RESULTS AND DISCUSSION}

The morphology of the iron oxide nanoparticles under study was characterized locally and globally using transmission electron microscopy (TEM) and small-angle x-ray and neutron scattering (SAXS/SANS), respectively. TEM images as presented in Fig. 1(a) reveal cubic nanoparticles of 8.6(6) nm edge length. Due to the rotational average of the dispersed nanocubes and for simplification of the more complex refinements of the SANS data, the small-angle scattering data were refined primarily by spherical form factors and conversion into cubic scales is performed via the radius of gyration. According to the different scattering length density contrasts between the nondeuterated oleic acid ligand shell and the deuterated toluene matrix for $\mathrm{x}$ rays and neutrons [Fig. 1(b)], the SAXS data was refined using a spherical particle form factor, whereas the SANS data enabled refinement of the full core-shell form factor, i.e., including the oleic acid ligand shell. Both probes consistently lead to an inorganic nanoparticle radius of $R_{S}=5.43(1) \mathrm{nm}$ and a narrow log normal size distribution of $8.2(2) \%$ FWHM. The ligand shell thickness was determined as 1.43(2) nm. The obtained spherical particle radius $R_{S}$ can be related to the cubic particle edge length $a_{c}$ via the radius of gyration $R_{G}$ according to $R_{G}^{2}=3 / 5 R_{S}^{2}=1 / 4 a_{c}^{2}$, 


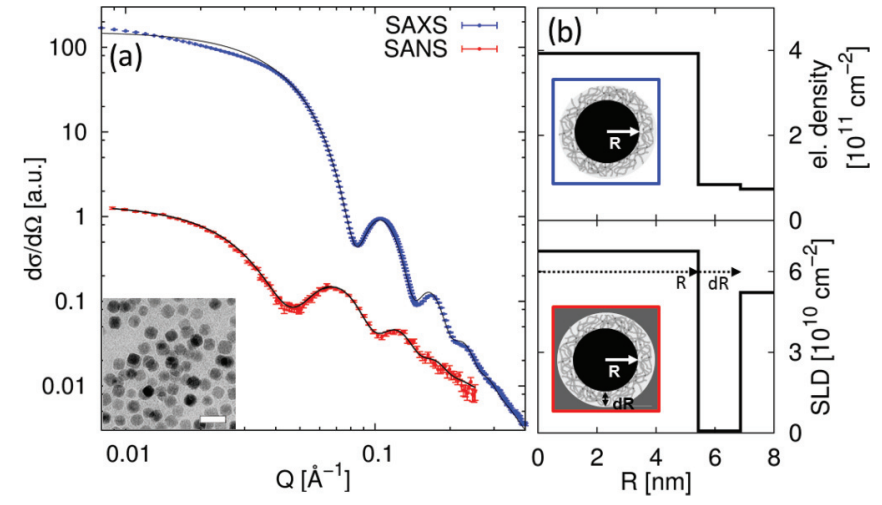

FIG. 1. (Color online) Morphology of iron oxide nanocubes. (a) The SAXS and SANS by nanocubes dispersed in deuterated toluene were refined according to a spherical form factor model. Inset: TEM image (scale bar $=20 \mathrm{~nm}$ ). (b) Scattering length density profiles for $\mathrm{x}$ rays (top) and neutrons (bottom); the relative contrasts are depicted as insets.

yielding a particle edge length of $a_{c}=8.42(2) \mathrm{nm}$, which is in excellent agreement with the TEM results. Since the refined spherical particle size distribution partially accommodates the nonspherical particle shape of the nanocubes, it is regarded as the upper limit of the cubic edge length distribution. Temperature dependent magnetization measurements after cooling in field and in zero field [Fig. 2(b)] reveal a superparamagnetic blocking temperature of 86(5) K. A Verwey transition has not been observed. Field dependent magnetization measurements [Fig. 2(a)] performed in the superparamagnetic phase (at $300 \mathrm{~K})$ exhibit the expected Langevin behavior with a saturation magnetization of $0.3 \frac{\mathrm{MA}}{\mathrm{m}}$. A particle magnetization of $16650(45) \mu_{\mathrm{B}}$ is extracted from the data along with a magnetic particle volume of 508(1) $\mathrm{nm}^{3}$, which are both in excellent agreement with our recent polarized SANS analysis [32].

Wide-angle $\mathrm{x}$-ray and neutron diffraction experiments confirm the maghemite/magnetite $\left(\gamma-\mathrm{Fe}_{2} \mathrm{O}_{3} / \mathrm{Fe}_{3} \mathrm{O}_{4}\right)$ spinel structure of the nanoparticles [Figs. 2(d) and 2(e)]. The cubic lattice parameter determined by Le Bail refinement is consistent for XRD $[a=8.356(1) \AA]$ and neutron diffraction $[a=8.358(1) \AA]$ data and indicates the maghemite atomic structure $(8.348 \AA$ [33]) rather than magnetite $(8.39 \AA$ [34]). This is further supported by analysis of the atomic pair distribution function and the neutron scattering length density obtained from SANS $[32,35]$. For evaluation of the magnetic scattering contribution in maghemite nanoparticles, neutron diffraction with full $x y z$ polarization analysis was carried out on a similar nanoparticle sample of the same composition but larger particle size of $14.6 \mathrm{~nm}$ in diameter. The separation into nuclear coherent, incoherent, and magnetic scattering contributions confirms the largely magnetic origin of the (111) reflection in maghemite at $1.31 \AA^{-1}$ [Fig. 2(c)]. Accordingly,
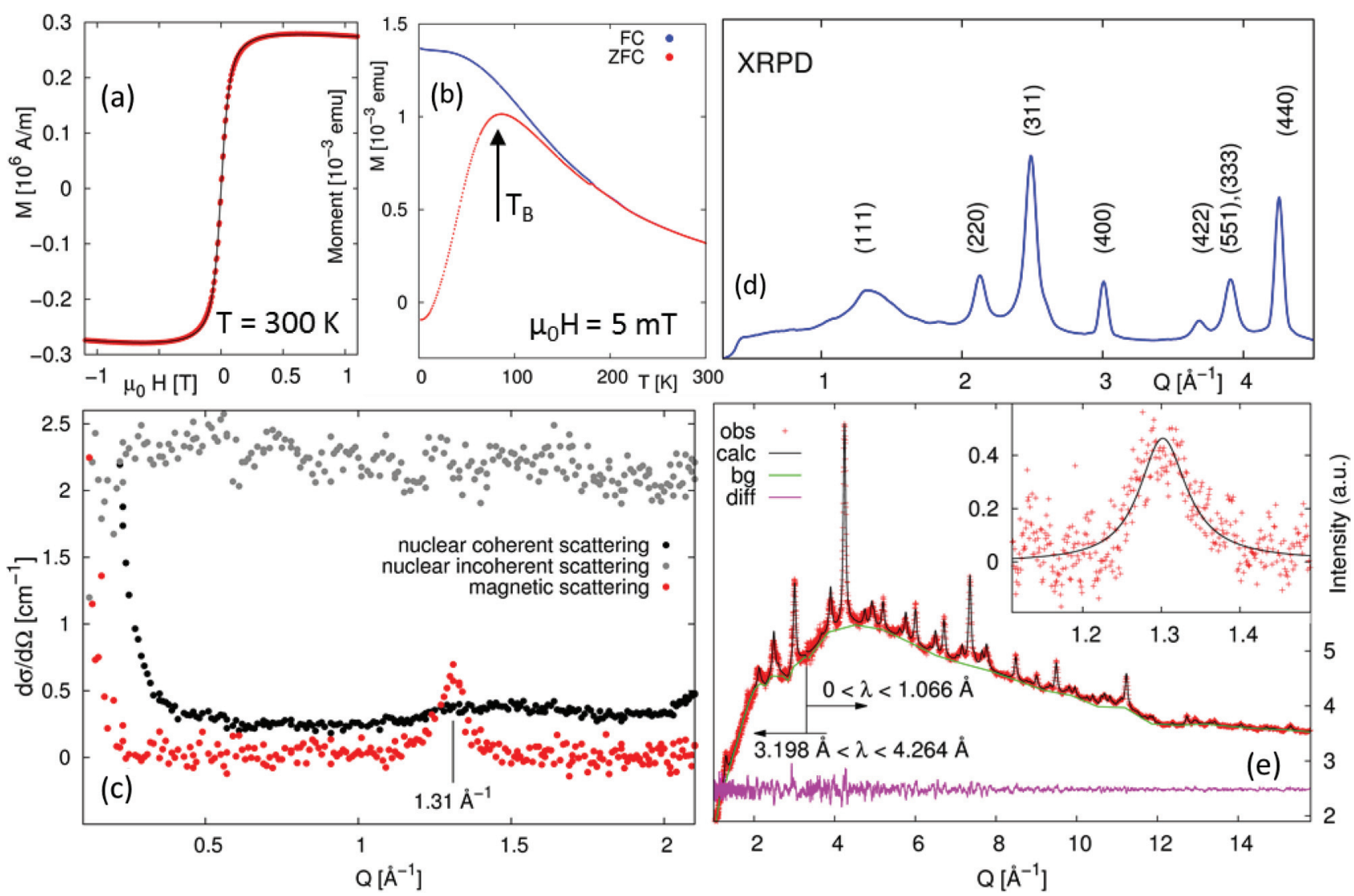

FIG. 2. (Color online) Magnetization and diffraction data. (a) Field dependent magnetization data. (b) Temperature dependent magnetization data. (c) Separation of nuclear and magnetic neutron scattering cross sections using neutron diffraction with polarization analysis. (d) Synchrotron XRPD by the nanoparticle powder. (e) TOF neutron diffraction and Le Bail refinement for two wavelength bands. Inset: profile of the (111) reflection after background subtraction. 


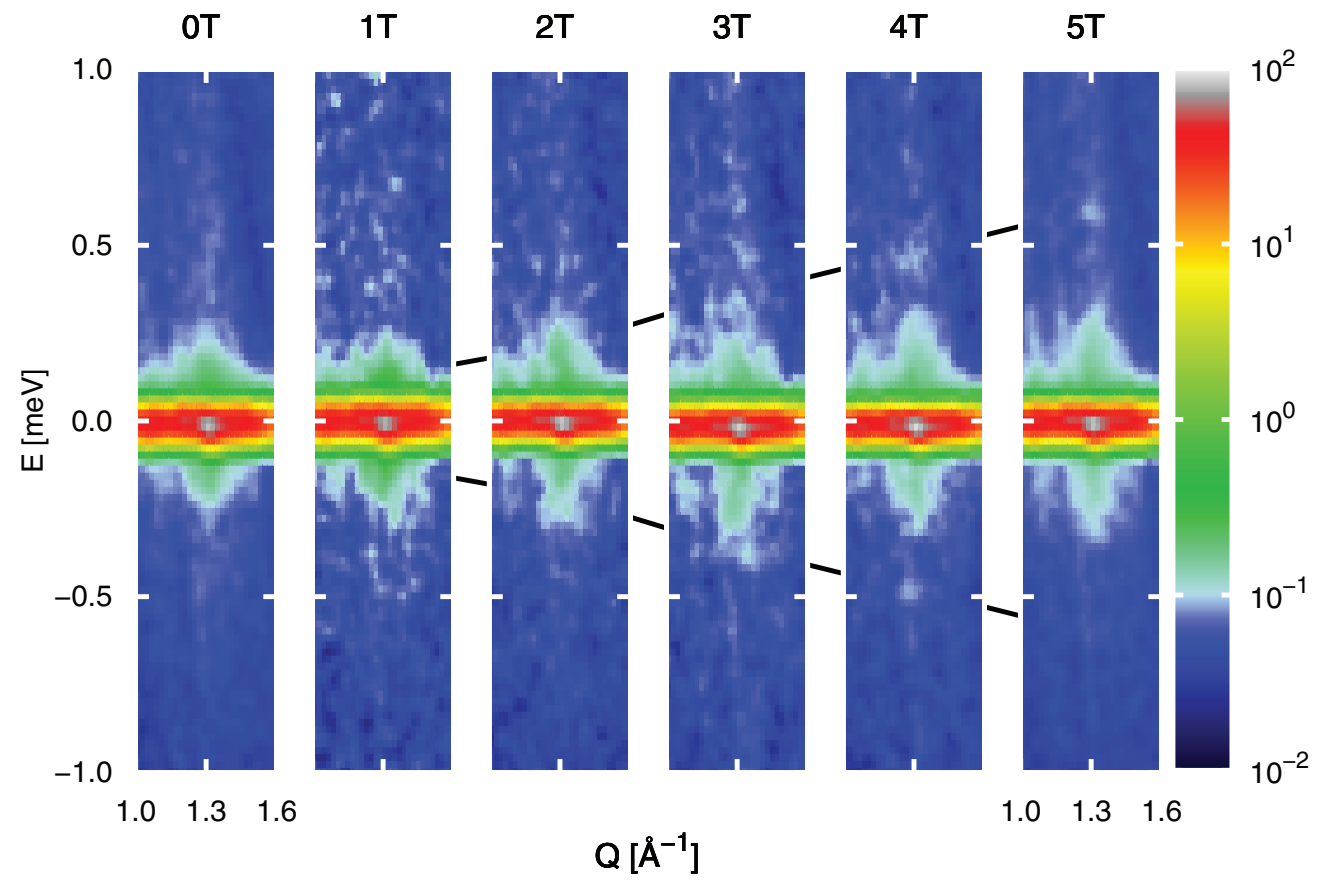

FIG. 3. (Color online) Inelastic neutron scattering by iron oxide nanocubes. Scattering data measured in different applied magnetic fields in the vicinity of the (111) magnetic reflection is presented $(T=100 \mathrm{~K})$.

spin wave excitations were studied using inelastic neutron scattering around this (111) reflection.

Elastic line scans of the original sample studied at the Cold Neutron Chopper Spectrometer (CNCS), SNS, confirm the $Q$ position of the (111) reflection at $1.34 \AA^{-1}$ and reveal a constant elastic scattering intensity for different applied magnetic fields. Color plots of the inelastic neutron scattering intensity in the range of $1.0 \AA^{-1}$ are shown in Fig. 3 . In particular in a high applied magnetic field, a magnetic excitation is recognized as field dependent inelastic scattering peaks with an energy transfer increasing up to $0.5 \mathrm{meV}(4 \mathrm{~T})$ and $0.6 \mathrm{meV}(5 \mathrm{~T})$. In a lower applied magnetic field, these peaks merge into the quasielastic scattering background of the elastic line and can hardly be distinguished. Energy projection at constant $Q\left(1.24-1.36 \AA^{-1}\right)$ allows for refinement of these inelastic satellite peaks and their energy transfer [Fig. 4(a)]. For analysis of the field dependence of the energy transfer, we followed the approximation of the average precession frequency in the presence of an external field $B_{\text {ext }}$ [13]:

$$
\hbar \omega \approx g \mu_{B} \sqrt{B_{A, \mathrm{eff}}^{2}+B_{\mathrm{ext}}^{2}},
$$

with $g$ the Lande factor, $\mu_{\mathrm{B}}$ the Bohr magneton, and $B_{A \text {,eff }}$ the effective anisotropy field. The anisotropy field $B_{A}$ is related to the anisotropy constant $K$ and the saturation magnetization per unit volume $M$ by

$$
B_{A}=\frac{2 K}{M},
$$

whereas the effective anisotropy field $B_{A \text {,eff }}$ is related to both magnetization sublattices in ferrimagnetic maghemite by

$$
B_{A, \mathrm{eff}}=\frac{B_{A 1} M_{1}+B_{A 2} M_{2}}{M_{1}-M_{2}} .
$$

This approximation is made on the basis of antiparallel orientation of the two ferrimagnetic sublattices with equal Landé factor as is valid for maghemite [13]. The field dependence of the energy transfer is refined using Eq. (1), leading to an effective anisotropy field $B_{A \text {,eff }}=0.45(16) \mathrm{T}$ and a Landé factor $g=2.05(2)$ [Fig. 4(b)]. The obtained Landé factor is in excellent agreement with $g=2$ expected for the spin-only $\mathrm{Fe}^{3+}$ ions as present in maghemite. The determined effective anisotropy field is slightly larger than reported for spherical maghemite nanoparticles of similar size in the past $\left[B_{A, \text { eff }}=0.3(1) \mathrm{T}\right]$ [13]. This increased anisotropy field observed here may be due to the anisotropic particle shape and the corresponding increased shape anisotropy.

The magnetic anisotropy constant $K$ is related to the effective anisotropy field $B_{A, \text { eff }}$ and the saturation magnetization according to $K=1 / 2 B_{A, \text { eff }} M_{s}$. Using the saturation magnetization of 3.04(1) $\times 10^{5} \frac{\mathrm{J}}{\mathrm{Tm}^{3}}$ obtained by macroscopic magnetization measurements and the anisotropy field determined above, we calculate an anisotropy constant of $(6.8 \pm 2.4) \times 10^{4} \frac{\mathrm{J}}{\mathrm{m}^{3}}$. Despite its large uncertainty (propagated through the large uncertainty of the effective anisotropy field), the obtained anisotropy constant is larger than reported for spherical maghemite nanoparticles of similar size as determined from magnetization measurements $\left(K \sim 1.5 \times 10^{4} \frac{\mathrm{J}}{\mathrm{m}^{3}}\right)$ [36], muon spin relaxation $\left[K=(1.3-4.2) \times 10^{4} \frac{\mathrm{J}}{\mathrm{m}^{3}}\right]$, and Mössbauer measurements $\left[K=(2.3-3.3) \times 10^{4} \frac{\mathrm{J}}{\mathrm{m}^{3}}\right]$ [37]. It is furthermore significantly larger than reported for bulk maghemite $\left(K=4.7 \times 10^{3} \frac{\mathrm{J}}{\mathrm{m}^{3}}\right)$ [38]. Calculation of the magnetic anisotropy constant from macroscopic magnetization data follows the approximation $K V_{\mathrm{mag}} / k_{B} T_{B} \approx 25$, corresponding to an experimental measuring time of $\tau=10 \mathrm{~s}$ [37]. Using the macroscopically measured blocking temperature of $T_{B}=86(5) \mathrm{K}$ and the magnetic particle volume of $V_{\mathrm{mag}}=$ 

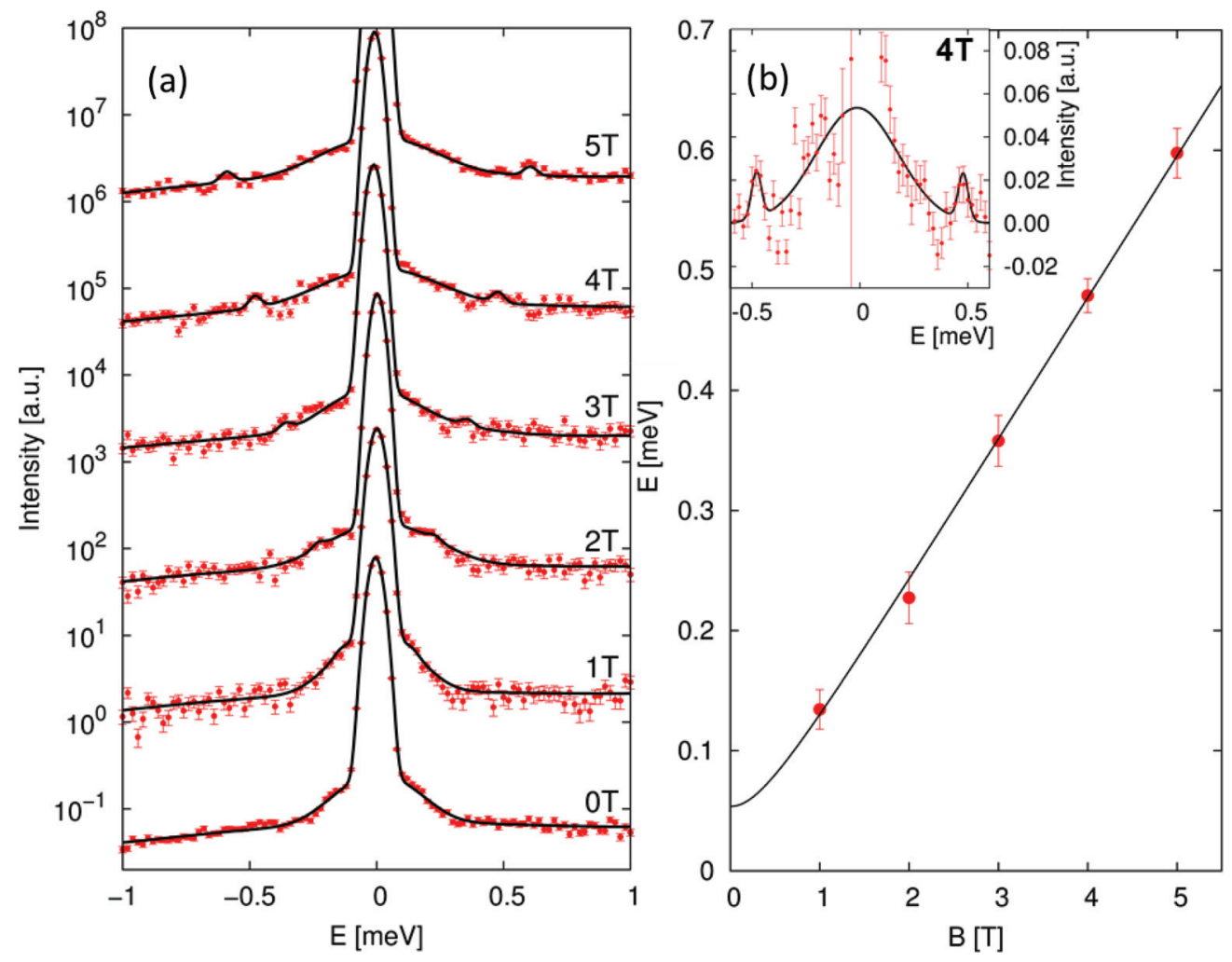

FIG. 4. (Color online) (a) Projections of the inelastic scattering data presented in Fig. 3 with refinement of the field dependent satellite reflections. (b) Field dependence of the determined satellite energy. Inset: data set after subtraction of the background scattering contribution and elastic line.

$508(1) \mathrm{nm}^{3}$ we obtain $K=5.8(3) \times 10^{4} \frac{\mathrm{J}}{\mathrm{m}^{3}}$, which is in good agreement with our inelastic neutron scattering results.

The agreement between macroscopic magnetization and inelastic neutron scattering results justifies the description of the observed inelastic scattering peaks as precession of the particle moment instead of alternative types of magnetic excitations. Whereas excitations of spin wave modes within the individual nanoparticles would be expected at larger energy transfer than studied here, excitations resulting from the dipolar coupling between individual nanoparticle spins will be heavily overdamped and occur at much lower energy transfers. An indication of such modes (heavily damped due to the disordered nanoparticle arrangement) may qualitatively be inferred from the field dependence of the quasielastic scattering contribution in Fig. 4(a).

A schematic comparing the energy scales of superparamagnetic relaxation and collective magnetic excitations as determined in this study is given in Fig. 5. Using the magnetic particle volume $V_{\text {mag }}=508(1) \mathrm{nm}^{3}$ and the anisotropy constant $K=6.8 \times 10^{4} \frac{\mathrm{J}}{\mathrm{m}^{3}}$, the superparamagnetic relaxation energy barrier in the studied sample is estimated as $K V_{\text {mag }}=0.216 \mathrm{eV}$. This is in good agreement with the superparamagnetic blocking temperature of 86(5) K observed using zero-field-cooled magnetization measurements. The total number of precession states distributed within this energy barrier is deduced from the integral nanoparticle moment of $\mu=16650(45) \mu_{\mathrm{B}}$, corresponding to $S=8325$ and $N=$ $2 S+1=16651$ states, which are divided equally into the two magnetization directions. This leads to an average density of precession states of $38.6 \mathrm{meV}^{-1}$. The lowest density of precession states near the energy minima is derived from the excitation energy determined by time-of-flight neutron spectroscopy ( $\Delta E=0.0534 \mathrm{meV}$ ) as $18.7 \mathrm{meV}^{-1}$, leading to the quadratic energy distribution of the precession states shown in Fig. 5. Weighting with the Bose factor at $100 \mathrm{~K}$ illustrates that only the lowest energy levels corresponding to

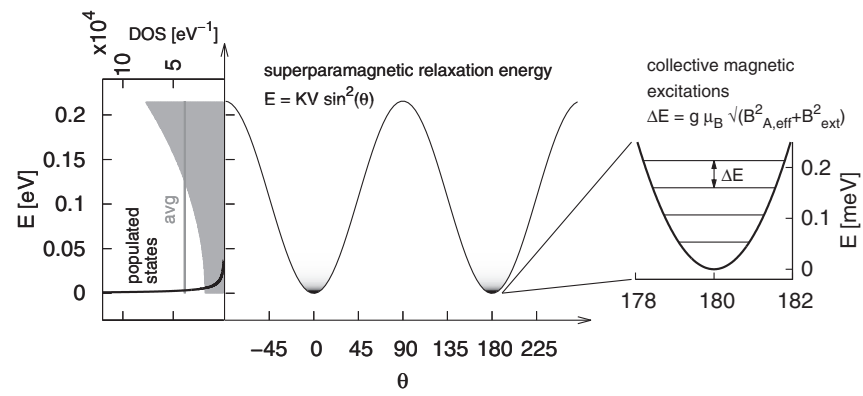

FIG. 5. Schematic representation of superparamagnetic relaxation and collective magnetic excitation energies in nanoparticles. For the graph, uniaxial anisotropy according to the Stoner-Wohlfarth model and vanishing external magnetic field were assumed for simplicity. The excitation energy of the uniform precession states is presented in dependence of the angle $\theta$ between nanoparticle spin and local easy axis. The gray scale reflects the relative occupancy of energy levels at $100 \mathrm{~K}$. The density of precession states and the populated states obtained by the Bose-factor weighting are given on the left. 
the uniform precession states are populated at the experiment conditions well below the superparamagnetic transition. In a classical picture, these states correspond to a precession of the total nanoparticle moments around the local anisotropy axis (or the local field axis which, for simplicity, is assumed to be collinear to the anisotropy axis). As the low lying states are equidistant, we observe sharp local excitations in our inelastic neutron spectroscopy study. Their excitation energies are about three orders of magnitude smaller compared to the energy barrier for superparamagnetic relaxation.

This illustrates that in a comparable amount of exposure time significant results can be obtained using either triple-axis or time-of-flight spectroscopies. Even in this case of rather localized excitations in wave-vector-energy space, modern chopper spectrometers compare favorably with triple axis spectrometers, since the lower incident flux is compensated by the larger reciprocal space coverage. The clear advantage of time-of-flight experiments as carried out here is in the extra information gained in each single exposure: elastic scattering information, a larger $Q$ range, and access to multiple excitations at different momentum, if existing.

\section{CONCLUSION}

We have studied the collective magnetic excitations in monodisperse cubic maghemite nanoparticles using time-offlight neutron spectroscopy. Our results are in good agreement with previous studies using triple-axis neutron spectroscopy [13], yielding a Landé factor of $g=2.05(2)$.

The determined large anisotropy field and the consequential large magnetic anisotropy are in good agreement with macroscopic magnetization measurements and may be attributed to enhanced shape anisotropy due to the cubic shape of the nanoparticles. The enhanced anisotropy is helpful, but still not sufficient to make these particles of interest for information technology. However, combined with additional effects, such as exchange coupling to an antiferromagnetic substrate, the blocking temperature might be pushed into a region relevant for applications.

Finally, the density of uniform precession states derived in this study in combination with the superparamagnetic energy barrier determined by macroscopic and microscopic magnetization measurements provides a consistent view of the energy scales of superparamagnetic relaxation and collective magnetic excitations in magnetic nanoparticles.

\section{ACKNOWLEDGMENTS}

We acknowledge HASYLAB/DESY and the Argonne Photon Source (APS) for providing the synchrotron radiation facilities at beamline B1 and 6-ID-D, respectively. Institut Laue-Langevin (ILL) and JCNS are acknowledged for provision of neutron scattering facilities at the D22 and DNS instruments, respectively. Research conducted at SNS (POWGEN and CNCS instruments) was sponsored by the Scientific User Facilities Division, Office of Basic Energy Sciences, US Department of Energy. We acknowledge Dr. U. Vainio, Dr. D. Robinson, Dr. A. Wiedenmann, Dr. Y. Su, and Dr. O. Gourdon for their support in data acquisition at these instruments. E.W., G.S.A., and L.B. acknowledge the Swedish Research Council (VR) for financial support. S.D. acknowledges funding by the 7 th European Community Framework Programme (PIEF-GA-2011-298918).
[1] M. E. McHenry and D. E. Laughlin, Acta Mater. 48, 223 (2000).

[2] A. P. Alivisatos, Science 271, 933 (1996).

[3] F. E. Kruis, H. Fissan, and A. Peled, J. Aerosol Sci. 29, 511 (1998).

[4] K. Raj and R. Moskowitz, J. Magn. Magn. Mater. 85, 233 (1990).

[5] Q. A. Pankhurst, J. Connolly, S. K. Jones, and J. Dobson, J. Phys. D: Appl. Phys. 36, R167 (2003).

[6] S. Bader, Rev. Mod. Phys. 78, 1 (2006).

[7] Y. Gossuin, S. Disch, Q. L. Vuong, P. Gillis, R. P. Hermann, J. H. Park, and M. Sailor, Contrast Media Mol. Imaging 5, 318 (2010).

[8] Y. Gossuin, A. Hocq, Q. L. Vuong, S. Disch, R. P. Hermann, and P. Gillis, Nanotechnology 19, 475102 (2008).

[9] S. Mørup, Appl. Phys. 11, 63 (1976).

[10] S. Mørup and B. R. Hansen, Phys. Rev. B 72, 024418 (2005).

[11] S. Mørup, J. Magn. Magn. Mater. 37, 39 (1983).

[12] J. van Lierop and D. H. Ryan, Phys. Rev. B 63, 064406 (2001).

[13] K. Lefmann, F. Bødker, S. N. Klausen, M. F. Hansen, K. N. Clausen, P.-A. Lindgård, and S. Mørup, Europhys. Lett. 54, 526 (2001).

[14] M. F. Hansen, F. Bødker, S. Mørup, K. Lefmann, K. N. Clausen, and P.-A. Lindgård, Phys. Rev. Lett. 79, 4910 (1997).

[15] M. F. Hansen, F. Bødker, S. Mørup, K. Lefmann, K. N. Clausen, and P.-A. Lindgård, J. Magn. Magn. Mater. 221, 10 (2000).
[16] S. N. Klausen, K. Lefmann, P.-A. Lindgård, L. Theil Kuhn, C. R. H. Bahl, C. Frandsen, S. Mørup, B. Roessli, N. Cavadini, and C. Niedermayer, Phys. Rev. B 70, 214411 (2004).

[17] L. Theil Kuhn, K. Lefmann, S. N. Klausen, H. M. Rønnow, A. Murani, and R. Stewart, Physica B 350, E217 (2004).

[18] K. Lefmann, F. Bødker, M. F. Hansen, H. Vázquez, N. B. Christensen, P.-A. Lindgård, K. N. Clausen, and S. Mørup, Eur. Phys. J. D 9, 491 (1999).

[19] M. Hennion, C. Bellouard, I. Mirebeau, J. L. Dormann, and M. Nogues, Europhys. Lett. 25, 43 (1994).

[20] M. Feygenson, X. Teng, S. E. Inderhees, Y. Yiu, W. Du, W. Han, J. Wen, Z. Xu, A. A. Podlesnyak, J. L. Niedziela, M. Hagen, Y. Qiu, C. M. Brown, L. Zhang, and M. C. Aronson, Phys. Rev. B 83, 174414 (2011).

[21] A. Ahniyaz, Y. Sakamoto, and L. Bergström, Proc. Natl. Acad. Sci. USA 104, 17570 (2007).

[22] Q. Song and Z. J. Zhang, J. Am. Chem. Soc. 126, 6164 (2004).

[23] G. Salazar-Alvarez, J. Qin, V. Šepelák, I. Bergmann, M. Vasilakaki, K. N. Trohidou, J. D. Ardisson, W. A. A. Macedo, M. Mikhaylova, M. Muhammed, M. D. Baró, and J. Nogués, J. Am. Chem. Soc. 130, 13234 (2008).

[24] J. Park, K. An, Y. Hwang, J. Park, H. Noh, J. Kim, J. Park, N. Hwang, and T. Hyeon, Nat. Mater. 3, 891 (2004). 
[25] T. Hyeon, S. Seong Lee, J. Park, Y. Chung, and H. Bin Na, J. Am. Chem. Soc. 123, 12798 (2001).

[26] J. Park, E. Lee, N. M. Hwang, M. Kang, S. C. Kim, Y. Hwang, J. G. Park, H. J. Noh, J. Y. Kom, J. H. Park, and T. Hyeon, Ang. Chem., Int. Ed. 44, 2872 (2005).

[27] A. Huq, J. P. Hodges, O. Gourdon, and L. Heroux, Z. Kristallogr. Proc. 1, 127 (2011).

[28] A. C. Larson and R. B. Von Dreele, Los Alamos National Laboratory Report LAUR No. 86, 1994 (unpublished).

[29] B. H. Toby, J. Appl. Crystallogr. 34, 210 (2001).

[30] G. Ehlers, A. A. Podlesnyak, J. L. Niedziela, E. B. Iverson, and P. E. Sokol, Rev. Sci. Instrum. 82, 085108 (2011).

[31] http://www.mantidproject.org.
[32] S. Disch, E. Wetterskog, R. P. Hermann, A. Wiedenmann, U. Vainio, G. Salazar-Alvarez, L. Bergström, and T. Brückel, New J. Phys. 14, 013025 (2012).

[33] C. Greaves, J. Solid State Chem. 49, 325 (1983).

[34] R. Cornell and U. Schwertmann, The Iron Oxides: Structure, Properties, Reactions, Occurrences and Uses (John Wiley \& Sons, Ltd., New York, 2006).

[35] S. Disch et al. (unpublished).

[36] T. Jonsson, J. Mattson, P. Nordblad, and P. Svedlindh, J. Magn. Magn. Mater. 168, 269 (1997).

[37] L. Rebbouh, R. P. Hermann, F. Grandjean, T. Hyeon, K. An, A. Amato, and G. J. Long, Phys. Rev. B 76, 174422 (2007).

[38] J. B. Birks, Proc. Phys. Soc. 63, 65 (1950). 